\title{
GHOST IMAGES IN A UNIVERSE WITH \\ INHOMOGENEOUS MASS DISTRIBUTION
}

\author{
J. M. BARNOTHY \\ 833 Lincoln Street, Evanston, Ill., U.S.A. \\ and \\ M. F. BARNOTHY \\ University of Illinois, Chicago, Ill., U.S.A.
}

\begin{abstract}
An attempt is made to explain Morgan cD supergiant galaxies, the microwave background radiation and extended discrete radio sources as ghost images in a universe of strong positive curvature and inhomogeneous mass distribution.
\end{abstract}

The observations of Humason et al. (1956), and of Minkowski (1960) and Baum (1962) revealed that up to $z=0.46$ the Hubble plot of galaxies follows the equation

$$
\mathrm{B}=5 \log z+23.23 \pm 0.13 \text {. }
$$

The first order regression line of the redshift-apparent magnitude relation of 161 QSOs with known $z$ and B value has the form (Barnothy and Barnothy, 1969)

$$
\mathrm{B}=5 \log z-3 \log z+17.85 \pm 0.08
$$

if Grewing's (1968) $K$-corrections are applied to the B magnitudes. Figure 1 is the histogram of 182 QSOs as a function of their V magnitudes, in five redshift intervals,

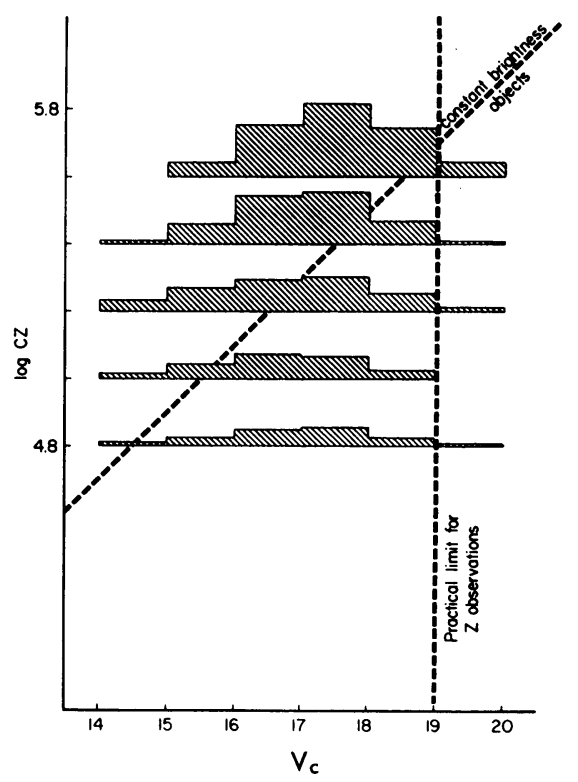

Fig. 1. Distribution of 182 quasi-stellar objects in five redshift intervals. The line with $45^{\circ}$ slope corresponds to the Hubble plot of objects of constant luminosity. 
from $\log c z=4.8$ to 5.8 . (The curves were smoothed using a $0.25(1+2+1)$ sliding average.) As we can see, the slope of the regression line through the center of gravity of the histograms, or through the peaks of the histograms is closer to a vertical line than to the $45^{\circ}$ line, corresponding to a Hubble plot of constant average luminosity objects in a Euclidean space or in a standard model $\left(q_{0}=\sigma_{0}=1\right)$ universe. The peak in the histogram of the highest redshift interval is still well to the left from the practical limit of redshift determinations $(\mathrm{V}=+19)$, implying that the steepness of the Hubble plot of QSOs is not the result of observational selection effects.

The first term in Equations (1) and (2) is the expression of the inverse distance square law of geometrial optics. The second term, missing in (1), indicates that the average luminosity of galaxies is constant, while the luminosity of QSOs increases as $z^{1.2}$. The difference in the constant terms indicates that at $z=1$ the average luminosity of a QSO would be 5.4 mag. higher than that of a galaxy. The difference in the two terms of the Hubble plots represents two primary characteristics of QSOs, which are not shared by other known astronomical objects. We have shown that in certain model universes (FIB and some M1-6 type models) where the space has a strong positive curvature, one can quantitatively explain the second term in the Hubble plot of QSOs, provided that the majority of QSOs are gravitational lens images.

Three dimensional spaces of positive curvature have a focusing effect on electromagnetic waves emitted by a source, the focal point representing the antipode of the source. Should our universe correspond to a type with such properties, then the observation of ghost images of galaxies, or of proto-galaxies may become possible, provided that the age of the universe is sufficiently long to allow the formation of ghost images.

The FIB model cosmology is particularly suited to produce observable images. Partly because its space has the metric of a projective space, in which every point is its own antipode, partly because the luminosity distances are by a factor of $1+z$ smaller than in expanding universes; and finally because the life time of an FIB universe is on the average many times larger than 41 billion years, the time needed by a photon to complete a roundtrip. We would like to mention three well known astronomical phenomena: Morgan's cD supergiant galaxies, the microwave background radiation and the extended radio sources, which all three may be produced by ghost images. Let us first obtain some insight into the position the FIB model occupies relative to other more commonly accepted models, such as the Big Bang and the steady-state model.

The Big Bang cosmology was conceived by Gamow. All the different models of the Big Bang family correspond to solutions of the field equations of the general theory of relativity, and are based on three assumptions: (a) that there existed once an immensely dense neutron gas of about $10^{14} \mathrm{~K}$ temperature; (b) that this neutron gas was since eternity, for reasons one cannot explain with present physical laws, in a 'frozen' state; and (c) that about 5 to 7 billion years ago this metaphysical state ceased to exist with the consequence that the neutron gas started to expand thereafter following present physical laws. The cosmological redshift is in these cosmologies a Doppler 
effect caused by the expansion. The Big Bang cosmologies do not conform to the perfect cosmological principle.

The principal event, the ceasing of the metaphysical state could by its very nature occur but once. But even this happened such a long time ago that any investigation of this event, or of the metaphysical state preceding it, is impossible. It has to be accepted as an 'act of God', outside of the realm of scientific inquiry and reasoning.

Sandage has shown that a model of the type $q_{0}=\sigma_{0}=1$ and $\Lambda=0$ is up to $z=0.46$ in good agreement with the Hubble plot of galaxies. However, with zero cosmological constant, the general theory of relativity would come into contradiction with Mach's principle (Horak, 1970). Furthermore a density parameter $\sigma_{0}=1$ corresponds to $\varrho_{0}=2 \times 10^{-29} \mathrm{~g} \mathrm{~cm}^{-3}$, a density which is 28 times higher than the density of visible matter in space.

In the steady-state cosmology of Bondi and Gold (1948) the cosmological redshift is similarly caused through the expansion of the universe. But here the expansion took place since an infinite past and is explained by the continuous creation of matter, the appearance of nucleons in empty space at a rate of $5 \times 10^{-47} \mathrm{~g} \mathrm{~cm}^{-3} \mathrm{~s}^{-1}$, or $10^{51}$ nucleons $\mathrm{Mpc}^{-3} \mathrm{~s}^{-1}$. The steady-state cosmology agrees with Mach's principle and with the perfect cosmological principle. It rejects, however, the field equations of the general relativity theory. The space is assumed to be Euclidean and infinite.

The basic assumption of this cosmology, the appearance of nucleons in empty space is in contradiction to the law of conservation of matter and energy as it is observed in the appearance of elementary particles in nuclear physical experiments. It also contradicts the commonly accepted concept that in a volume limited by impermeable walls energy and matter have to be conserved. The law of conservation of energy and of matter was for this reason reformulated to fit the theory. It was postulated that energy and matter are conserved in a volume, the walls of which permit the escape of matter equivalent in amount to the amount of matter appearing through the process of continuous creation in the said volume. While in other expanding cosmologies energy and matter are conserved in co-moving coordinate volumes, in a steadystate model only the density of matter and of energy, but not the total energy, is conserved.

The creation of nucleons in empty space also contradicts the observation that a new elementary particle, or particle-anti-particle pair can appear only in the presence of already existing particles and then only if the energy-momentum transfer is of the order of the Compton wavelength of the appearing particle. The theory does not clarify whether during the continuous creation of matter lepton and baryon conservation laws are satisfied, or when a nucleon and antinucleon are created at the same place, why do they not become instantaneously annihilated, whereby the released energy would increase the radiation density in the universe to a degree which would contradict observations.

McCrea (1964) suggested, partly to overcome the above mentioned difficulties, that matter is created at places where matter is already present (nucleons in galaxies, antinucleons in antigalaxies) at a rate proportional to the density of the matter, or 
$4 \times 10^{6}$ nucleons $\mathrm{g}^{-1} \mathrm{~s}^{-1}$. This variant of the steady-state cosmology needs an additional assumption, namely that matter appears in the form of atoms. If matter should appear in the form of free neutrons, then through neutron capture radioactive isotopes would form and scintillation detectors, for example, should show a $10^{5}$ times higher background than actually observed.

According to Bondi (1960) the great value of the steady-state cosmology is that "the problem of creation is brought within the scope of physical inquiry and is examined in detail instead of, as in other theories, being handed over to metaphysics" (l.c. p. 140). This is, however, contradicted later by the statement "It is clear that it is utterly impossible to observe directly such a rate of creation. There is therefore no contradiction whatever with the observations"' (l.c. p. 143).

The FIB cosmology (Barnothy and Forro, 1944) was originally developed to explain a phenomenon we found between 1928 and 1942 while registering with large coincidence telescopes the sidereal time periodicity of cosmic radiation. This anisotropy of cosmic radiation is a Doppler effect caused through the rotation of the Galaxy. Its existence was predicted by Compton and Getting (1935) and it was discovered by Hess in 1936. The FIB cosmology explains the flux density, the spectral characteristics and the extreme particle energies $\left(10^{21} \mathrm{eV}\right)$ of cosmic rays arriving from outside of our Galaxy (Barnothy and Barnothy, 1963, 1967a).

The metric of the FIB universe is that of Einstein's static solution of the field equations. It conforms with Mach's principle and with the perfect cosmological principle, nevertheless it does not prevent a galactic evolution. (See Appendix.)

The fundamental assumption of the FIB theory is that photons (and elementary particles of zero rest mass) while travelling along geodesics, change their energy and wavelength relative to the units of stable elementary particles. The cosmological redshift can be expressed as a function of the distance of the source $(D)$ if we use the radius of curvature of the space $(R)$ as the length unit.

$$
1+z=e^{D / R}
$$

It is our belief that any fundamental theory which can not propose an experimentum crucis, capable of proving the existence of the phenomenon postulated as its basic assumption, is nothing more than idle speculation. We have, therefore, already in 1947 proposed an experimentum crucis (Barnothy and Barnothy, 1963) which can be performed with standard observatory equipment: 40-in. telescope, diffraction grating and photomultiplier. We definitely disagree with Bondi's opinion (l.c.) that it is to the advantage of a theory if the postulated basic phenomenon eludes observations and thus can never be disproven.

Equation (3) connects the redshift with the radius of curvature $R$, figuring in the field equations. Because the space of the Einstein static solution is finite, the value of the Hubble constant depends solely on the total mass of the universe. Accepting Eddington's suggestion that the number of nucleons in the universe cannot exceed the number of independent wavefunctions, we obtain $H=73 \mathrm{~km} \mathrm{~s}^{-1} \mathrm{Mpc}^{-1}$, a value within the limits of current determinations e. g. Sandage (1968) $75 \mathrm{~km} \mathrm{~s}^{-1} \mathrm{Mpc}^{-1}$. 
Through the focusing effect of the positively curved space, ghost images will appear at the location of the object itself. The redshift of the first, second $\ldots n$-th images is

$$
z=e^{n \pi}-1=23.14^{n}-1
$$

The bolometric luminosity of the successive ghost images remains equal to that of the object at the time of emission, provided that we can disregard absorption during the propagation of the electromagnetic radiation through the universe. The photons will pass during their round trips in the vicinity of galaxies and be deflected in the gravitational fields of the latter. The superposition of many small deflections produces a de-focusing, blurring effect, with the result that the ghost image of a point source will appear as a slightly elliptical diffraction disk, which has a diameter equal to or larger than that of giant galaxies (Barnothy and Forro, 1944). The surface brightness distribution of the ghost images will resemble a normal distribution, the standard deviation $\theta$ of a photon from the direction of the geodesics along which it started to move is

$$
\theta=\frac{4 M G}{c^{2} r}(1.6+\ln N)^{1 / 2}
$$

where $M$ is the average mass and $N$ the total number of galaxies affecting the scattering, $r$ the average closest approach of a photon to a galaxy. In a FIB universe the average density of matter in space is $7 \times 10^{-30} \mathrm{~g} \mathrm{~cm}^{-3}$, ten times higher than the observed density of visible matter. In accordance with Peebles (1969) we assume that this missing mass is made up from dead galaxies. The computations give that $\theta \sim 1$ arc sec, the radius of the first ghost image is $\Delta \sim 30 \mathrm{kpc}$ and the radius of the $n$-th ghost image is

$$
\Delta_{n}=(n)^{1 / 2} 30 \mathrm{kpc}
$$

Let us now discuss some astrophysical phenomena which could be produced by ghost images.

\section{A. MORGAN CD TYPE SUPERGIANT GALAXIES}

Ambartsumian (1958) was the first to propose an explosive origin of galaxies. Recently Serrsic (1970) has investigated this hypothesis anew. His model describing the properties of galaxies and of clusters of galaxies resulting from fragmentation of proto-galaxies is shown to be in fairly good agreement with observations. Should this theory be correct then the massive proto-galaxy does not exist any more; merely its fragments, the galaxies of the cluster, can be seen. Since the assumed implosion of the proto-galaxy, preceding the fragmentation, produces a considerable amount of energy, the temperature must have been at that time very high and the proto-galaxy very luminous. Should this event have occurred earlier than the time needed for a complete round trip, then the first ghost image of the proto-galaxy should be observable now near the center of the cluster of galaxies, created through its fragmentation.

Morgan and Lesh (1965) have carried out a survey of all galaxy clusters in Abell's 
catalogue having richness greater than 2 . Of the 85 clusters examined, 22 were found to have dominating supergiant galaxies of the class $\mathrm{cD}$ in Morgan's classification. The main morphological characteristics of $\mathrm{cD}$ galaxies are: they are centrally located in the cluster, of which they are outstandingly the largest and brightest member; they are never highly flattened in shape; they have a nucleus surrounded by a very extended amorphous envelope; the nucleus has a lower surface brightness than the nuclei of the other larger members of the cluster and finally at lower exposure the nucleus might split up into 2 or 3 components.

The supergiant galaxy NGC 6166 in Abell's cluster 2199, can be considered as a prototype of cD galaxies. It was investigated by Minkowski (1961) and by E. M. Burbidge (1962). The nucleus of this system is composed of three (or perhaps four components), the size, luminosity and spectrum of which would be quite normal for medium size elliptical galaxies. The three components are positioned at the apices of a triangle of side lengths $12.5,8.5$ and $6.0 \mathrm{arc} \mathrm{sec}$, respectively. The phenomenon which qualifies this system to be recognized as a Morgan $\mathrm{cD}$ supergiant galaxy, is the existence of a very extended envelope or 'background' of very high luminosity, the brightness of which increases toward its center, but the degree of its central condensation is remarkably low and although at first glance it may look like a supergiant elliptical galaxy, the bright nuclear region typical of types E and S0 is absent. This 'background' seems to be centered on one of the components, transforming this component into the brightest one. (No. 1 component in Minkowski's and $A$ component in Burbidge's notation). The diameter of this background to the isophote 25 mag arc $\mathrm{sec}^{-2}$ is 150 arc sec, or $91 \mathrm{kpc}$; it has a reddish color with $\mathrm{B}-\mathrm{V}=+0.85$ and its absolute luminosity is $-22.01 \mathrm{mag}$.

The cluster is at a distance of $121 \mathrm{Mpc}$ from us. Should the luminous 'background' be the ghost image of the proto-galaxy prior to its fragmentation, then, according to Equation (6), the diameter of its nucleus should be $60 \mathrm{kpc}$; its brightness could be explained if the temperature of the plasma surrounding the proto-galaxy were of the order of 50000 to $100000 \mathrm{~K}$ and thus emitted a sufficient amount of light in the wavelength range of $200 \AA-300 \AA$ to be now, redshifted by $z=22$, observable in the visible region. We should expect, however, that one would find the greatest part of the optical radiation in the red and infrared regions. This is born out by the observation that when using a Kodak IIa-0 emulsion with Schott UG-1 filter and Ross corrector (Burbidge, 1962), the 'background' vanished, leaving only a blue semi-stellar nucleus near its central region. Nevertheless a galaxy must obviously be still present at the place of the first component, otherwise this component could not yield emission and absorption lines with the same redshift value as found in the other components.

\section{B. MICROWAVE BACKGROUND RADIATION}

The existence of a microwave background radiation has now been established through observations at many radioastronomical observatories. It was found that in the range from 20.7 to $0.26 \mathrm{~cm}$ wavelength the flux density corresponds to a black body radiation of $2.7 \pm 0.3 \mathrm{~K}$. Gamow et al. twenty years ago predicted the existence of a 
microwave background radiation of $5 \mathrm{~K}$ as being the remnant of the initial flash of the Big Bang. Dicke et al. (1965) have accepted Gamow's explanation for the microwave background radiation (actually the Princeton group was unaware of Gamow's work and rediscovered the primeval fireball). Many scientists consider the microwave background radiation as a definite proof of the Big Bang event. In our opinion to extrapolate from a phenomenon with observed temperature $3 \mathrm{~K}$, to an event of $10^{14} \mathrm{~K}$ temperature, which occurred 4 to 7 billion years ago and to consider this explanation as a proof is of questionable value.

As mentioned, in an FIB universe the antipode of a galaxy is the galaxy itself. We are therefore situated within the ghost images of the Galaxy, and the combined radiation of the latter will simulate for us a background radiation. Provided that the galaxy did not move appreciably away from its original position during the time needed for the round trips of its light, we may be close to the center of these ghost images, rendering the radiation fairly isotropic. A slight asymmetry should however exist as a consequence of the Compton-Getting effect, in the same manner as this was observed in cosmic radiation.

If there were no redshift, the situation would be similar to that which would exist if all of the stars of the Galaxy were now re-distributed in the much larger volume of its image. Knowing the luminosity of the Galaxy $\left(\mathrm{L}=5 \times 10^{43} \mathrm{erg} \mathrm{s}^{-1}\right)$ and the brightness distribution of the image, we may compute the total flux of the electromagnetic waves arriving at the center of the first ghost image to

$$
F=L / 4 \pi \Delta^{2}=4 \times 10^{-4} \mathrm{erg} \mathrm{s}^{-1} \mathrm{~cm}^{-1} \mathrm{ster}^{-1}
$$

Because the successive ghost images have an $n^{1 / 2}$ larger radius, the flux at the center of the $n$-th image will be

$$
F_{n}=F n^{-1}
$$

The optical continuum of the Galaxy is fairly well approximated by that of a black body of $6000 \mathrm{~K}$ temperature. The ratio of the total flux at the center of an image to the total flux inside of a black body of $6000 \mathrm{~K}$ yields the fraction $C$ of the sky which would now be covered by stars of $6000 \mathrm{~K}$.

$$
C_{n}=F_{n} / 6000^{4} \sigma
$$

where $\sigma=5.67 \times 10^{-5} \mathrm{erg} \mathrm{deg}^{-4} \mathrm{~cm}^{-2} \mathrm{~s}^{-1}$ is the Stefan-Boltzmann constant. The brightness temperature is computed by radioastronomers from the equation

$$
T_{b}=I_{v} \lambda^{2} / 2 k
$$

$T_{b}$ is a linear function of the flux density $I_{v}$, observed within a $1 \mathrm{~Hz}$ band width at wavelength $\lambda$ and $k$ is the Boltzmann constant.

Without redshift we would observe a brightness temperature, which is by a factor $C / n$ lower than $6000 \mathrm{~K}$, provided that we remain on the long-wave tail of the spectrum where the Rayleigh-Jeans law is still valid. On account of the redshift suffered by the photons during their round trips, we have to apply a correction, taking care of the difference between emitted and observed wavelengths; furthermore we have to apply 
the non-selective $K$-correction. Our final result is then that the brightness temperature produced by the $n$-th ghost image is

$$
T_{b}=\frac{F}{6000^{3}} \frac{e^{n \pi(2-i)}}{\sigma n}
$$

where $i$ is the average spectral index of extended radio sources $(i \sim-0.8) . T_{b}=3.7 \mathrm{~K}$ for the third ghost image. This is in agreement with the observed temperature of the microwave background radiation. Should the fourth ghost image of the Galaxy already exist, its brightness temperature would be $1.4 \times 10^{4} \mathrm{~K}$. Although the background radiation of the fourth image would be shifted into the meter range, it should still be well observable. The observed brightness temperature in the meter wavelength range is, however, merely a few hundred degrees Kelvin. We conclude therefore, that the Galaxy is more than 123 billion years old but cannot be older than 164 billion years. This age is not too long for a galaxy in a universe where the gas content of the galaxies is continuously replenished through the dispersion of the matter of old stars and of the matter of the nuclei of the galaxies following impacts of high energy neutrinos. (See Appendix.)

\section{EXTENDED RADIO SOURCES}

The successive ghost images of a galaxy are like snapshots of the galaxy taken at intervals of 41 billion years. As we have seen, the images are blurred and somewhat smeared out through the gravitational scattering of light on intervening galaxies and each image is redshifted by a factor $e^{\pi}=23.14$ relative to the preceding image. The wavelength range commonly used by radio observatories is covered by the fourth ghost image. But the third image starts to come in below $10 \mathrm{~cm}$ wavelength and the fifth image at wavelengths longer than $1 \mathrm{~m}$. Should matter be completely homogeneously distributed in space so that the curvature of space is exactly the same everywhere, then all successive images would appear at the same place: at the place of the object. In reality space curvature is not exactly constant, the focal length of the cosmological lens is not the same in all directions, consequently the location of the ghost images will not always coincide with that of the source. Even numbered images among them and odd numbered images among them should, however, more closely overlap.

Should the galaxy serving as the source have a proper motion, the successive images will appear at different locations. Taking $100 \mathrm{~km} \mathrm{~s}^{-1}$ as the average velocity of this proper motion and assuming that the direction of this motion does not change, the successive images would form a chain of radio sources, displaced by about $4 \mathrm{Mpc}$ relative to each other.

In reality, during the 41 billion years needed for each round trip, the direction of the proper motion will most probably change many times. Consequently, one would expect that the successive ghost images would be distributed around the optical galaxy (should the latter still exist) at distances which are merely a fraction of $4 \mathrm{Mpc}$, say, of the order of $300 \mathrm{kpc}$. This agrees well with the observations. 
If the narrow light beam which supplies the luminosity of the ghost image in the direction of the observer passes near a very heavy galaxy an effect may occur which resembles the effect of a gravitational lens: two separate images will be produced from the same source. The separation however is in most instances less than the apparent diameter of the image. This effect may be repeated, resulting in ghost images with 2 to 3 nuclei. The overall diameter of a ghost image complex with several nuclei, would not be considerably larger than that following from Equation (6). We come, therefore to the conclusion that the spatial distance $(\sim 300-1000 \mathrm{kpc})$ and the diameter of the images $(\sim 60-120 \mathrm{kpc})$ will remain roughly unchanged. Thus the apparent spacing and apparent size of the components of a double radio source will depend on the distance of the radio source from the observer with the ratio of size to separation $(\sim 1: 5)$ remaining unchanged.

It should be emphasized that the compact radio sources found in QSOs are of entirely different origin (Barnothy and Barnothy, 1966) from the extended radio sources discussed here. Should a QSO be a gravitational lens image, the extended radio sources surrounding it may be ghost images of the deflector galaxy and not of the galaxy serving as object of the lens.

Through the non-selective $K$-correction the observed flux density of each successive ghost image is by $1+z=23.14$ higher than that of the preceding image. When all three images are present, the overlapping spectra will produce a flat spectrum which simulates a synchrotron radiation spectrum having an $i=-1$ spectral index (see Figure 2), in good agreement with the average spectral index of radio sources being about -0.8 . If the fifth image is missing, the radio source will display a strongly

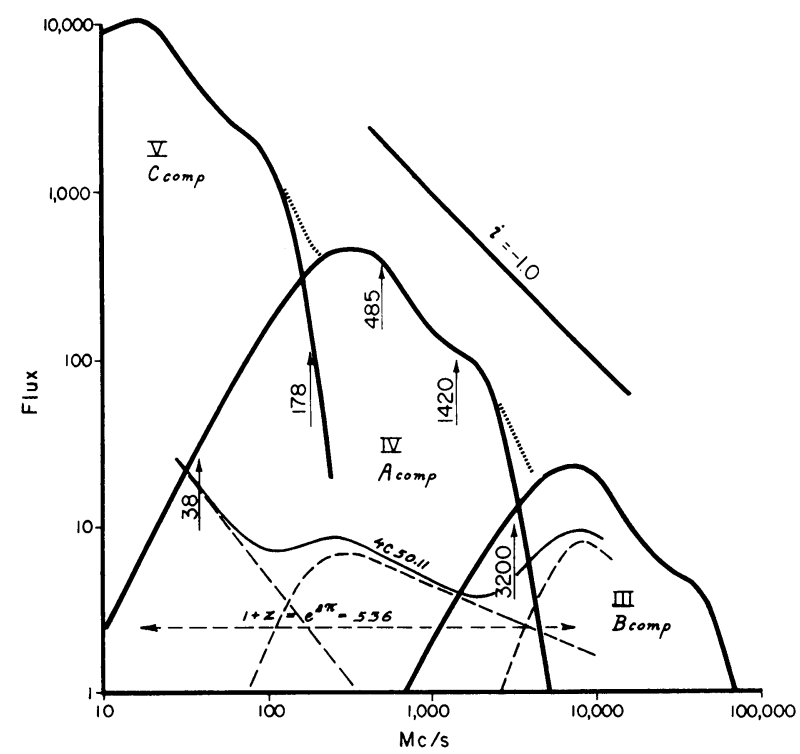

Fig. 2. The spectrum of the third, fourth and fifth ghost image of a galaxy. The lower line is the spectrum of the radio source $4 \mathrm{C} 50.11$. 
curved spectrum. Whenever the three images are spatially well separated, each of them will represent a discrete radio source, displaying the original black body spectrum of the galaxy from which they originate. Observing at a wavelength where the spectra of two successive images overlap, the spectral index will be strongly positive in the lower order and strongly negative in the higher order image.

The lower line in Figure 2 is the spectrum of the radio source $4 \mathrm{C} \mathrm{50.11.} \mathrm{The} \mathrm{three}$ components are well separated, the spectral peaks of the components are shifted, as expected, by a frequency ratio of approximately 23 relative to each other.

The spectral index of $4 \mathrm{C} 50.11$ is about -0.2 , five times flatter than the theoretically expected value of -1.0 . The difference could be interpreted as being caused by an increase in the intrinsic brightness of the galaxy by $\frac{1}{2}$ mag. every 10 billion years.

\section{Appendix}

The world is populated by two different kinds of entities: those which have a rest mass (elementary particles and their combinations) and those which have not: signal carriers such as photons, neutrinos and gravitons.

The main difference between these two populations is that elementary particles have inherent characteristics (rest mass, charge, spin), the value of which can be determined through repeated measurements to any desired accuracy. All stable elementary particles have characteristic quantities which have the same numerical value for all members of the same species, or are in a constant ratio between different species. Thus these characteristic quantities determine a mass-length-time unit system, which constitutes the basis of all our measurements. In what follows we shall call this unit system the substratum. Signal carriers, on the other hand, have merely individual characteristics, which, without introducing radical changes through the measuring process, can be determined but once. The accuracy of any single determination is subject to Heisenberg's uncertainty relation. This circumstance renders energy, momentum and angular momentum conservation laws invalid for individual signal carriers. The conservation laws when applied to signal carriers have only a statistical validity in the sense that the total energy, momentum and angular momentum carried in a beam remains constant within the statistical error limits.

The statistical nature of the conservation laws of signal carriers allows the characteristic quantities of signal carriers to undergo changes relative to the substratum or even permits that signal carriers disappear from the beam, provided that these changes do not alter the total energy, the total momentum and the total angular momentum carried in the beam.

The FIB theory, like all fundamental theories, sets out from a number of postulates, which themselves are not further explainable. Some of these postulates are common with other cosmological theories, others are expressions of well established observations in the form of a physical law, while those which are new permit an experimental verification.

The first postulate is the perfect cosmological principle: the world presents the same 
aspect everywhere at all times. This postulate is supported by astronomical observations and follows from Mach's principle and the conservation laws. It brings all physical phenomena into the realm of physical investigations and insures the continuity of physical laws.

The second postulate: the universe can be described by the field equations of the general theory of relativity, and Mach's principle and the spatial symmetry principle are valid. This postulate restricts the cosmological solutions to the static Einstein solution of general theory of relativity, that is to a closed non-expanding hypersphere embedded in a four-dimensional continuum. A hypersphere of spherical geometry would enclose a four-dimensional volume, the two sides of our three-dimensional space would not be alike: one would be 'inside', while the other 'outside', violating the perfect spatial symmetry principle. We infer therefore, that the space of the universe has elliptical geometry where the antipode of every point is identical with the point itself.

The third postulate: elementary particles or photons, can only appear in interactions with existing elementary particles; the reciprocal value of the energy momentum transfer being thereby of the same order of magnitude as the Compton wavelength of the appearing particle, is merely an expression of the experimental fact that the appearance of elementary particles and of photons is always observed under circumstances specified above. The third postulate could be also called the 'genetic code' principle, which seems to represent a quite general law, applicable to all creation events producing new entities.

The fourth postulate expresses the fundamental tenet of the theory, the FIB phenomenon: the characteristic quantities of signal carriers change relative to the substratum as a function of the time elapsed since their emission, whereby the absolute value of the numerical change in the energy or relativistic mass of the signal carrier is equivalent to a change which the energy of the signal carrier would suffer while moving toward or away from a mass $M$ situated at a distance $R$, (where $M$ is the total mass of the universe and $R$ its radius of curvature.)

Any increase or decrease in the energy of signal carriers after emission relative to the substratum would entail a violation of the conservation of energy, assumed to be statistically valid for the total energy in a beam of signal carriers, unless new signal carriers would be created, or existing signal carriers would disappear from the beam, depending whether the direction of the change is a decrease or an increase in the energy, respectively. The creation of new signal carriers in empty space is prohibited by the third postulate, while no such restriction exists for the disappearance of a signal carrier. From this we can infer that the direction of the energy change of a signal carrier can only be an increase in their energy. The energy change and the disappearance of signal carriers are connected through the conservation of energy principle and are mutual pre-requisites for each other. To conserve the total energy of a beam, the function governing the disappearance has to be reciprocal to the function governing the energy change of the signal carriers. The fourth postulate does not specify the form of the function but one can mathematically derive that in a 
steady state universe, such as the FIB universe, the function has to be an exponential function.

As was shown (Barnothy and Barnothy, 1963), a consequent application of the above postulates and of the conservation laws leads to the inference that if $n_{0}$ photons of wavelength $\lambda_{0}$ and energy $E_{0}$ are emitted by a source and have travelled for a time $t$ before reaching the observer, the beam would contain at arrival $n$ photons of wavelength $\lambda$ and energy $E$, where

$$
n=n_{0} e^{-t c / R} ; \lambda=\lambda_{0} e^{t c / R}=\lambda_{0} e^{t H} ; \quad E=E_{0} e^{t c / R}
$$

where $H$ is the Hubble constant.

In a closed universe signal carriers return to the source after one or more round trips. In an FIB universe, however, instead of the emitted large number of low energy photons, merely a small number of high energy photons will return. The free energy degraded in thermodynamical processes is upgraded through the FIB phenomenon and returned in a low entropy energy form, which after absorption in the substratum, can be used anew to maintain the flow of natural processes. The total entropy of the universe remains constant, the universe representing thus a perpetuum mobile of the second order, without contradicting thereby the second law of thermodynamics.

The transformation of matter into radiation is taking place in the stars and in the nuclei of galaxies and remains on the average constant as long as the nuclear fuel is not exhausted. The rate of transformation of radiant energy into matter depends however, on the energy of the signal carrier. As it is well known, the Einstein static universe is in a labile equilibrium: a slight decrease in radiation pressure would start an accelerated continuous expansion, and vice versa. However, even the slightest expansion process would, through a Doppler effect, decrease the energy of the returning signal carriers and lower the probability of transferring their energy into matter. Considering now the constant transformation rate of matter into radiant energy, this in turn would entail an increase in the density of radiant energy and thus a slowing down and eventually a reversal of the previously started expansion. The FIB phenomenon represents thus a regulating mechanism which maintains the equilibrium of an Einstein static universe.

The space of a FIB universe is filled with neutrino radiation, originating from the neutrino emission of stars. Its differential spectrum has the form (Barnothy and Barnothy, 1963)

$$
N(E)=3 \times 10^{-6} / E
$$

where $N$ ster $^{-1} \mathrm{~cm}^{-2} \mathrm{~s}^{-1}$ is the number of neutrinos emitted and $E$ their energy in ergs. When neutrinos pass through matter their gravitational field will interact with the particles of matter. Their total energy loss is proportional to the square of the neutrino energy and to the total surface density of the crossed mass. Low energy neutrinos may pass without interactions, but high energy neutrinos may lose their 
total energy while crossing a star or a nucleus of a galaxy. On the average the nucleus of a galaxy of $4 \mathrm{kpc}$ diameter will be hit in every 10 million years by a neutrino of a relativistic mass in excess of one $M_{\odot}$, and in every ten billion years by one which has a mass in excess of $1000 M_{\odot}$. The energy supplied through the impact of these high energy neutrinos could be the energy source which produces the high intensity infrared radiation, discovered by Low and co-workers. Due to the random character of the neutrino impacts, the infrared radiation produced should display a quite considerable intensity fluctuation and the sources of strong infrared radiation may have the properties of secondary nuclei within the nucleus of a galaxy. The neutrino impact could also supply an explanation of the fragmentation of protogalaxies into clusters of galaxies, of the phenomenon of supernovae and of meteor craters in which no traces of meteorites were found (Barnothy and Barnothy, 1967a).

From Equation (12) it follows that both wavelength and energy of photons increase simultaneously, while their number decreases. The same equations are valid also for other signal carriers, hence it is quite generally true that the information uncertainty conveyed by all signal carriers increases with the distance and that Heisenberg's uncertainty relation has the form

$$
\Delta p \cdot \Delta q=s e^{2 t H}
$$

with $s$ denoting the spin of stable elementary particles.

FIB is an acronym, expressing this Far-away Information Blurring.

\section{References}

Ambartsumian, V. A.: 1958, in 'Solvay Conf. Rep.', Stoops, Bruxelles.

Barnothy, J. and Forro, M.: 1944, Csillagaszati Lapok 7, 65.

Barnothy, J. M. and Barnothy, M. F.: 1963, Z. Phys. 176, 435.

Barnothy, J. M. and Barnothy, M. F.: 1967a, Astron. J. 72, 784.

Barnothy, J. M. and Barnothy, M. F.: 1967b, Astron. J. 72, 291.

Barnothy, J. M. and Barnothy, M. F.: 1969, Nature 222, 759.

Baum, W. A.: 1962, in G. C. McVittie (ed.), 'Problem of Extra-Galactic Research', IAU Symp. 15, 390.

Bondi, H.: 1960, Cosmology, Cambridge University Press.

Bondi, H. and Gold, T.: 1948, Monthly Notices Roy. Astron. Soc. 108, 252.

Burbidge, E. M.: 1962, Astrophys. J. 136, 1134.

Compton, A. H. and Getting, I. A.: 1935, Phys. Rev. 47, 817.

Dicke, R. H., Peebles, P. J. E., Roll, P. G., and Wilkinson, D. T.: 1965, Astrophys. J. 142, 414.

Gamow, G.: 1946, Phys. Rev. 70, 527.

Grewing, M. and Lamla, E.: 1968, Z. Astrophys. 68, 473.

Hess, V. F. and Grazidei, H. Th.: 1936, Terr. Magn. Atmosph. Electr., March.

Horak, Z.: 1970, Bull. Astron. Inst. Czech. 21, 96.

Humason, M. L., Mayall, N. U., and Sandage, A. R.: 1956. Astron. J. 61, 97.

McCrea, W. H.: 1964, Monthly Notices Roy. Astron. Soc. 128, 335.

Minkowski, R.: 1960, Astrophys. J. 132, 908.

Minkowski, R.: 1961, Astron. J. 66, 558.

Morgan, W. W. and Lesh, J. R.: 1965, Astrophys. J. 142, 1364.

Peebles, P. J. E.: 1968, Astrophys. J. Letters 154, L121.

Sandage, A.: 1958, Astrophys. J. 127, 513.

Sandage, A.: 1968, Astrophys. J. Letters 152, L149.

Sersic, J. L.: 1970, Bull. Astron. Inst. Csl. $21,92$. 


\section{Discussion}

Solheim: In a Lemaitre model with poles, we may also predict a ghost image in opposite directions in the sky. In this case we may expect to see nearly the same redshift, within a few tenths - which is quite different from the FIB prediction of a difference of about $z=22$. Furthermore, the time difference between two images will be $10^{9}-10^{10} \mathrm{yr}$, which makes it very unlikely that we may observe short-lived $\left(10^{6}-10^{7} \mathrm{yr}\right)$ objects like quasars in both directions at the same time. 